\title{
A study on evaluation of 3D virtual rabbit kidney models by multidetector computed tomography images
}

\author{
Emrullah Eken ${ }^{1}$, Özşen Çorumluoğlu², Yahya Paksoy ${ }^{3}$, Kamil Beşoluk ${ }^{1}$, İbrahim Kalaycı ${ }^{2}$ \\ ${ }^{1}$ Department of Anatomy, Faculty of Veterinary Medicine, University of Selçuk, Konya, Turkey \\ ${ }^{2}$ Department of Geodesy and Photogrammetry, Faculty of Architecture and Engineering, University of Selçuk, Konya, Turkey \\ ${ }^{3}$ Department of Radiology, Meram School of Medicine, University of Selçuk, Konya, Turkey
}

\begin{abstract}
Objectives: This study was performed to reveal biometric peculiarities of New Zealand rabbit kidneys by means of threedimensional (3D) reconstruction of multidetector computed tomography (MDCT) images.

Methods: Under general anaesthesia, the kidneys of eight rabbits of both sexes were scanned by high resolution imaging using a general diagnostic MDCT. The thoracic and lumbar vertebrae of the rabbit were used as reference structure to locate exact position of the kidneys in the rabbit body. Finally, landmarks of the kidneys were identified and labelled in the MDCT images and then 3D models were formed to analyse the locations, dimensions and volumes of the kidneys with respect to the vertebral column in transparently modelled body.

Results: The left and right kidney volumes are $9153.3 \mathrm{~mm}^{3}$ and $9761 \mathrm{~mm}^{3}$ respectively. The right kidney is approximately $9 \%$ longer, $22 \%$ wider and $27 \%$ thicker than the left kidney. The left kidney is $16 \%$ flatter than the right one.

Conclusion: The authors have proposed that the 3D reconstructed results of the MDCT images can contribute to the anatomical education and clinical applications.
\end{abstract}

Key words: CT imaging; three-dimensional reconstruction; morphometry; kidney; rabbit

Anatomy 2009; 3: 40-44, @ 2009 TSACA

\section{Introduction}

Rabbits are used in human and veterinary medicine as experimental animals. Most medical instruments are also applied to humans after being tested generally on some animals. ${ }^{1}$ Technical achievements and the strict application of accurate anatomical knowledge to surgical and clinical practices have added a new dimension to the diagnosis and the surgical or medical treatments of some diseases. It has been already known that computed tomography (CT) currently plays a dominant role in diagnosis and evaluation of many human diseases and human anatomic structure. Recent papers have also described the use of the CT in veterinary medicine., ${ }^{2,3}$ Before any tool can become an effective diagnostic modality, normal species - specific data must be characterized. Publication of clinically relevant CT anatomy is basic to effective utilization of this modality in veterinary medicine. ${ }^{4}$ The CT anatomy can be used not only in diagnostic procedures but also in many biometric researches, which constitutes an immense contribution to the evaluation of breeds. ${ }^{5,6}$

Previous anatomical descriptions on the rabbit kidneys were rather superficial. ${ }^{7,8}$ Moreover, as there is no study on normal CT anatomy of the rabbit, this study 
was focused on normal biometric measurement of the rabbit kidneys by the help of pseudo-3D displays created from the multidetector computed tomography (MDCT) images. The MDCT is a recent technologic advance that allows rapid volume cover speed combined with thinslice images to create a volume data set suitable for workstation analysis in two-dimensional axial display, multiplanar reformation, or three-dimensional reconstructed imaging. The data obtained from the MDCT are sent to a computer to reconstruct all of the individual "snapshots" into a cross-sectional image (slice) of the internal organs and tissues for each complete rotation of the source of $x$ rays. Pseudo-3D displays are also created from a stack of $2 \mathrm{D}$ images of a large number of these parallel planes as snapshots. ${ }^{9}$

Authors have suggested that for following studies reduced slice thickness is the most significant factor to obtain more detailed multiple three-dimensional reconstructive images, and that the results from this study can also shed light on surgical and anatomical investigations related to rabbit kidney.

\section{Materials and Methods}

A total of eight adult healthy New Zealand Rabbits of both sexes aged 1.5-2 years and weighing between 3.5 and $4 \mathrm{~kg}$ were used. Animals were intravenously anaesthetized with $5 \mathrm{mg} / \mathrm{kg}$ of ketamine- $\mathrm{HCl}$ (Ketamidor ${ }^{\mathrm{TM}}$ Richer Pharma AG, Wels, Austria) and $20 \mathrm{mg} / \mathrm{kg}$ propofol (Propofol ${ }^{\mathrm{TM}}$ amp., Fresenius Kabi, Austria). Under anaesthesia, in a prone position the animal body was scanned by high resolution imaging using a general diagnostic MDCT. The MDCT was performed with a 64-section CT system (Somatom Sensation 64; Siemens Medical Solutions, Forchheim, Germany). Scanning along the axial axis was performed by using the following parameters: physical detector collimation, $32 \times 0.6$ $\mathrm{mm}$; resulting section collimation, $64 \times 0.6 \mathrm{~mm}$; section thickness, $0.75 \mathrm{~mm}$ (increment, $0.7 \mathrm{~mm}$ ); gantry rotation time, 330 msec; kVp, 120; mA, 300; spatial resolution, $512 \times 512$ pixels with pixel spacing, $0.92 \times 0.92$ and radiometric resolution MONOCHROME2 which gives 16 bit gray level. Dose and scanning parameters have been performed by radiologists in Meram School of
Medicine, University of Selçuk, Konya, Turkey, on the basis of the standardized protocol which considers the documented scanning practices and the recent studies ${ }^{10,11}$ to generate optimum image quality while maintaining individual radiation exposure at the lowest level. The axial images obtained were then stored in DICOM format to transfer to a personal computer in which the $3 \mathrm{D}$ modelling software (3D-DOCTOR for Windows, Ay Tasarım Ltd., Ankara, Turkey, http://www.aytasarim.com) was set up. This study considered the manually corrected automated segmentation for $3 \mathrm{D}$ reconstruction of images as in the literature. ${ }^{12}$ The points that have been improperly positioned after automatic boundary segmentation were edited manually throughout an interactive boundary editing routine; therefore this segmentation is called as semi-automatic segmentation. Manual editing process takes 3 to 4 minutes per image. Semi-automatic segmentation was done by determining the kidney boundaries automatically first, then the points which were not correctly positioned on the kidney boundaries were edited point by point with a computer mouse. After manual editing was rechecked visually, all the corrected boundaries of the kidney surfaces were stacked and overlaid to reconstruct the $3 \mathrm{D}$ model of bones by $3 \mathrm{D}$ rendering component of the software.

\section{The average measures describing rabbit kidneys by the 3D virtual models}

Based on the 2D images of axial sections from the CT, renal average measurements of both sexes regarding locations, dimensions and volumes and illustrations were recorded and reconstructed in the $3 \mathrm{D}$ for the average rabbit body. Considering thoracic and lumbar vertebrae used as reference model, whose $3 \mathrm{D}$ reconstructed images were obtained by only automatic segmentation procedures on the 2D CT images, some space coordinates of both kidneys were also measured.

It has been proposed that both biometric perspectives and the $3 \mathrm{D}$ reconstruction technique performed in this work add a new dimension to the future studies on reconstructive studies. 


\section{Results}

Some slides among all slices of the rabbit body and related to the kidneys were used for the construction of the 3D virtual kidney models. In Figure 1, top part shows two CT slices located almost at the centre of each kidney's volumetric space. Measurements edited on the images are referred to the volumetric centre position of each kidney with respect to the corresponding centre of the vertebral canal appearing in the same slice. So, radial distances between these two volumetric centres are 33 $\mathrm{mm}$ and $45 \mathrm{~mm}$ according to right and left kidneys respectively. It can also be seen from the Figure 1 that both kidney centres are located vertically at $15 \mathrm{~mm}$ down with respect to their corresponding vertebral centres. It means that there is no offset between the kidney centres with respect to the corresponding vertebrae. Focusing onto the bottom part of Figure 1, it can be seen that radial distance is $75 \mathrm{~mm}$ between the kidney centres.

Contrary to that there is no vertical offset between the kidney centres, there is an offset at the extent of 40.2 $\mathrm{mm}$ along the backbone direction and the right kidney comes first than the left kidney according to the rabbit's head location.

Volume data of both kidneys were obtained from the CT images through the constructed 3D models. The left and right kidney volumes are therefore $9153.3 \mathrm{~mm}^{3}$ and $9761 \mathrm{~mm}^{3}$ respectively and the right kidney is about 7\% bigger than the left one. Figure 2 depicts side and front view of the $3 \mathrm{D}$ virtual right and left kidneys with length, width and thickness measurements, considering the extremist points of the right and left kidneys in the $3 \mathrm{D}$ models. As it is seen from the figure, the right kidney is approximately $9 \%$ longer, $22 \%$ wider and $27 \%$ thicker than the left kidney with respect to the dimensions of about $39.9 \mathrm{~mm}, 26 \mathrm{~mm}$ and $25.1 \mathrm{~mm}$ and of about 36.5 $\mathrm{mm}, 21.4 \mathrm{~mm}$ and $19.8 \mathrm{~mm}$ for the length, width and thickness of the right and left kidneys respectively. Flatness is $3 \%$ and $19 \%$ for the right and left kidneys, respectively. So, the left kidney is $16 \%$ flatter than the right one. The angle between the plane passing through both kidney centres and the transversal plane is foundas $28^{\circ} .19$.

\section{Discussion}

In this study, as the semi-automatic segmentation procedures on the 2D CT images make some incorrect label assignment, the manual editing has been also has been also added to the image processing procedure to show nearly absolute 3D images and geometrical measurements of the rabbit kidneys. Therefore, it can be said that the $3 \mathrm{D}$ reconstructed images and findings from this study reflect accurately the true anatomical properties of the kidneys. Based on the 3D geometrical kidney, plastic rabbit kidney models of real dimension can also be created. Moreover, as long as the medical imaging and photogrammetry reasonably approximate each other, it may

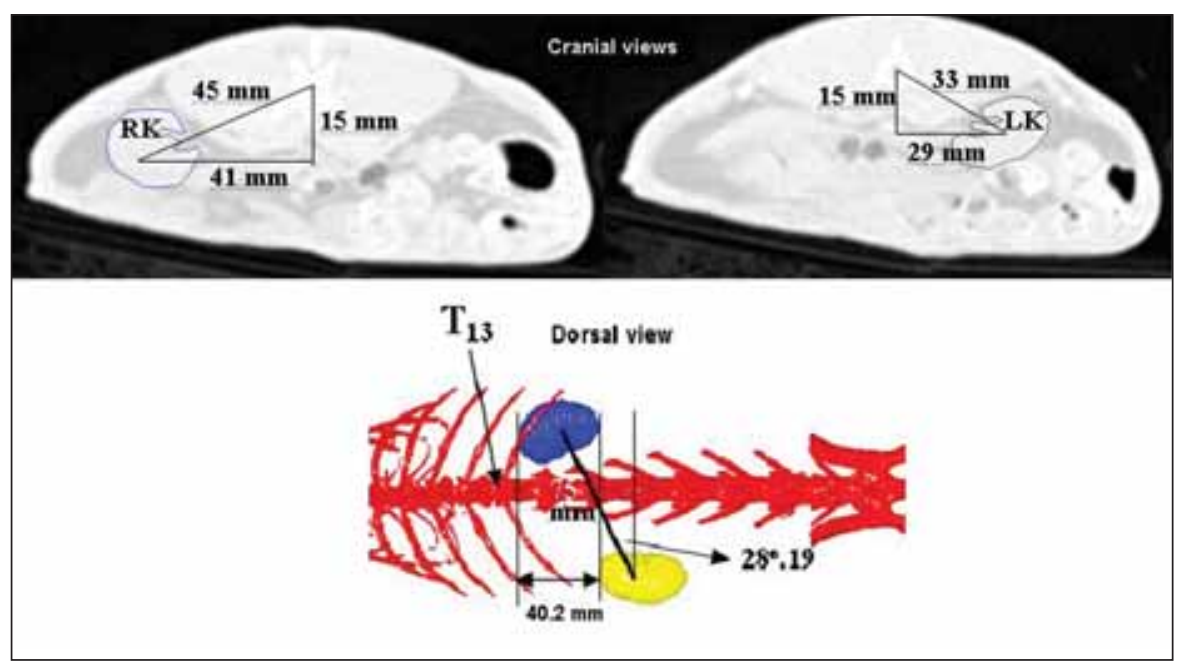

Figure 1. Measurements describing the location of rabbit kidneys with respect to one another. RK: right kidney; LK: left kidney. 
Figure 2. Dimensions of right and left rabbit kidneys. Length: cranial to caudal; Thickness: dorsal to ventral; Width: lateral to medial. Ca: caudal; Cr: cranial; D: dorsal; LK: left kidney; RK: right kidney; V: ventral.

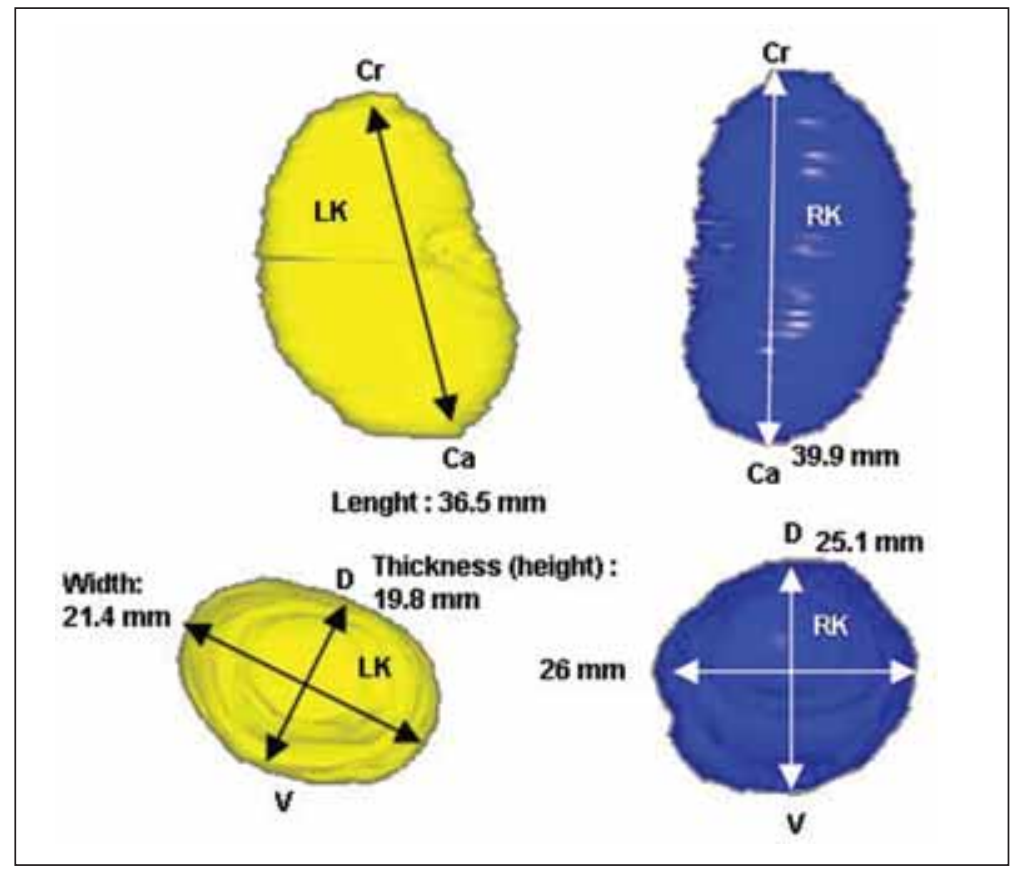

be possible to create new approaches for diagnostic and therapeutic procedures of the kidney related pathological disorders. It is true that this study have limitations depending on the manual editing, which introduces operator dependency and time consumption, and the technique may not be suitable for routine evaluation of the kidneys. However, in this study our main purpose has already been to provide basic morphometric information regarding the rabbit kidneys.

The authors showed that $3 \mathrm{D}$ modelling software (3D-DOCTOR) used in this study can be useful for obtaining various biometric measurements from reconstructed kidney models as the other softwares which were included in human reconstructive studies. ${ }^{13,14}$ Nevertheless, some renal size-related-measurements obtained from computerized tomography with 3D reconstruction may have not absolute accuracy enough to take into consideration in renal transplantations. ${ }^{15}$

In conclusion, the present study is the first to analyse the location, size and volume of rabbit kidneys in situ by $3 \mathrm{D}$ reconstruction of the $2 \mathrm{D}$ MDCT images. When considered point of medical view, the exact $3 \mathrm{D}$ reconstruction of a disease-suspected or evident anomalous region and its real time examination is very important.
Finally, parameters related to volume, shape of an anatomical structure including its relationships to critical regions can be determined thus being made an appropriate choice for treatment. The authors also suggest that this work adds a modern dimension to anatomical education.

\section{References}

1. Alfidi RJ, Macintyre WJ, Meaney TF, et al. Experimental studies to determine application of CAT scanning to the human body. Am f Roentgenol 1975; 124: 199-207.

2. Eken E, Gezici M. The influence of stomach volume on the liver topography in cats. Anat Histol Embryol 2002; 31: 99-104.

3. Kara M, Turan E, Dabanoglu I, Ocal MK. Computed tomographic assessment of the trachea in the German shepherd dog. Ann Anat 2004; 186: 317-21.

4. Smalwood JE, George TF. Anatomic atlas for computed tomography in the mesaticephalic dog: thorax and cranial abdomen. Vet Radiol Ultrasound 1993; 34: 65-84.

5. Regedon S, Franco A, Garin JM, Robina A Lignereux Y. Computerized tomographic determination of the cranial volume of the dog applied to racial and sexual differentiation. Acta Anat 1991; 142: 347-50.

6. Onar V, Kahvecioglu O, Cebi V. Computed tomographic analysis of the cranial cavity and neurocranium in the German shepherd dog (Alsatian) puppies. Vet Arbiv 2002; 72: 57-66. 
7. Barone R, Pavaux C, Blin PC, Cuo P. Atlas. D'anatomie du Lapin. Paris: Masson \& Cie; 1973.

8. McLaughlin CA, Chiasson RB. Laboratory Anatomy of the Rabbit. 3th ed. Dubuque: Wm. C. Brown Publishers; 1990.

9. Hu H, He HD, Foley WD, Fox SH. Four multidetector-row helical CT: Image quality and volume coverage speed. Radiology 2000; 215: 55-62.

10. Prokop M. General principles of MDCT. Eur 7 Radiol 2003; 45: S4-S10.

11. Kalra MK, Maher MM, Toth TL, Hamberg LM, Blake MA, Shepard J, Saini S. Strategies for CT radiation dose optimization. Radiology 2004; 230: 619-28.
12. Bazille A, Guttman MA, McVeigh ER, Zerhouni EA. Impact of semiautomated versus manual image segmentation errors on myocardial strain calculation by magnetic resonance tagging. Invest Radiol 1994; 29: 427-33.

13. Aldur MM. Creating computer aided 3D model of spleen and kidney based on Visible Human Project. Saudi Med 7 2005: 26: 51-6.

14. Turkvatan A, Ozdemir M, Cumhur T, Olcer T. Multidetector CT angiography of renal vasculature: normal anatomy and variants. Eur Radiol 2009: 19: 236-44.

15. Janoff DM, Davol P, Hazzard J, Lemmers MJ, Paduch DA, Barry JM. Computerized tomography with 3-dimensional reconstruction for the evaluation of renal size and arterial anatomy in the living kidney donor. 7 Urology 2004: 171: 27-30.

Correspondence to: Emrullah Eken, PhD

Department of Anatomy, Faculty of Veterinary Medicine, University of Selçuk, Campus

Konya 42079 Turkey

Phone: +90 33222336 17; Fax: +90 3322410063

e-mail: eeken@selcuk.edu.tr

Conflict of interest statement: No conflicts declared. 